\title{
Obturator hematoma following transobturator sling
}

\begin{abstract}
Background: This is a rare case of hematoma formation following suburethral sling placement via the transobturator approach.

Case: A 33-year-old woman presented with symptoms of urge and stress urinary incontinence. She underwent anterior and posterior colporrhaphy, and transobturator suburethral sling placement. She presented to the emergency room six days postoperatively with dizziness and mild vaginal bleeding. CT imaging revealed a 6.5 $\mathrm{x} 4.0 \times 4.7 \mathrm{~cm}$ hematoma in the left obturator region. Her symptoms subsided after conservative management. Subsequent follow-up visits showed improvement in her overall clinical status and no further intervention was required.
\end{abstract}

Conclusion: Patients with small postoperative hematomas who are clinically stable and asymptomatic may be managed with appropriate pain management and imaging surveillance.
Volume 9 Issue 6 - 2018

\author{
Sangeeta Ramani,' Bogdan A Grigorescu, ' \\ Gregory Ceus, ${ }^{2}$ George Lazarou' \\ 'Department of Obstetrics and Gynecology, NYU Winthrop \\ Hospital, USA \\ ${ }^{2}$ Albany Medical College, USA
}

\begin{abstract}
Correspondence: Sangeeta Ramani, MD, NYUWinthrop Hospital, Department of Obstetrics and Gynecology 259, First Street, Mineola, NY I I50I, USA, Tel (516) 663-9539, Fax (516)
\end{abstract} 742-7821, Email Samgeeta.ramani@nyulangone.org

Received: October 02, 2018 | Published: December 07, 2018

Keywords: obturator hematoma, suburethral sling, transobturator tape

\section{Case}

A 33-year-old female gravida 4 para 4 presented to the office with complaints of urge and stress urinary incontinence since her vaginal delivery four years ago. Stress symptoms were more bothersome than urge. Her past medical and surgical history were unremarkable. On physical exam, positive Q-tip test $>35^{\circ}$, POPQ stage 2 anterior vaginal prolapses consistent with midline cystocele, and POPQ stage 2 posterior vaginal prolapse consistent with rectocele were noted. Urodynamic testing revealed stress urinary incontinence with Valsalva at maximal capacity and mild detrusor overactivity. She failed conservative management with pelvic floor exercises and declined pessary management or anticholinergics.

The decision was made to proceed with anterior and posterior colporrhaphy, transobturator suburethral sling and cystoscopy. Our surgical approach was consistent with Delorme's description of the out-to-in technique. The skin over the medial aspects of the obturator foramens was incised and the helical introducers were guided out to in through the obturator foramens via the periurethral space. ${ }^{1}$ This space was previously dissected vaginally away from the obturator neurovascular bundle and away from the adductor group of muscles. The tail end of the metal introducers was brought to the vaginal area and periurethral spaces and were connected to the suburethral sling and reversed. Cystoscopy was performed with normal findings, and there was no obvious bleeding at the end of procedure.

Postoperatively, patient was found to have moderate vaginal bleeding with a small anterior vaginal hematoma. Patient was unable to pass active trial of void; therefore, foley was reinserted. Total estimated blood loss intraoperatively and postoperatively was $250 \mathrm{cc}$. Vital signs were stable, and hemoglobin/hematocrit was noted to be stable on repeat blood draws at 7.0/21.0. The patient was discharged on postoperative day 1 with a foley catheter.

The patient presented to the office on postoperative day 6 with symptoms of lightheadedness on ambulation and progressively worsening generalized weakness. She also reported abdominal discomfort and vaginal bleeding requiring 3-4 pads daily. Foley catheter was removed for trial of void. The patient was referred to the emergency room for imaging and further evaluation.

Pelvic examination revealed $30-50 \mathrm{cc}$ of blood in vaginal vault, with no active bleeding from vaginal mucosa and suture lines noted to be intact. Vital signs and labs were within normal limits other than hemoglobin/hematocrit $(8.1 / 23.5)$ noted to be stable since prior admission for the surgery. The patient underwent a computer tomography $(\mathrm{CT})$ scan of the abdomen and pelvis. Imaging revealed a hematoma in the left obturator musculature measuring $6.5 \times 4.0 \times$ $4.7 \mathrm{~cm}$ with mass effect on the bladder (Figure 1) (Figure 2). The patient was able to void without difficulty despite this mass effect. Given that vitals were stable, and her symptoms subsided after hydration and analgesia, decision was made to discharge patient with close outpatient follow-up. The patient was asymptomatic at subsequent visits 3-4 months following surgery, and no further intervention was required.

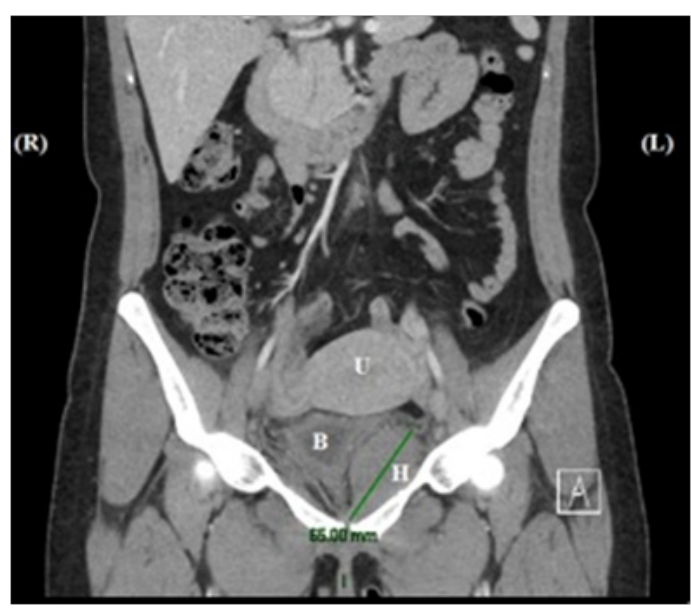

Figure I Coronal view of hematoma $(\mathrm{H})$ in the left obturator region measuring $6.5 \mathrm{~cm}$ in length with mass effect on the bladder (B), displacing it to the right. Uterus $(U)$ is superior to both the bladder and hematoma. $(R)$, right; (L), left. 


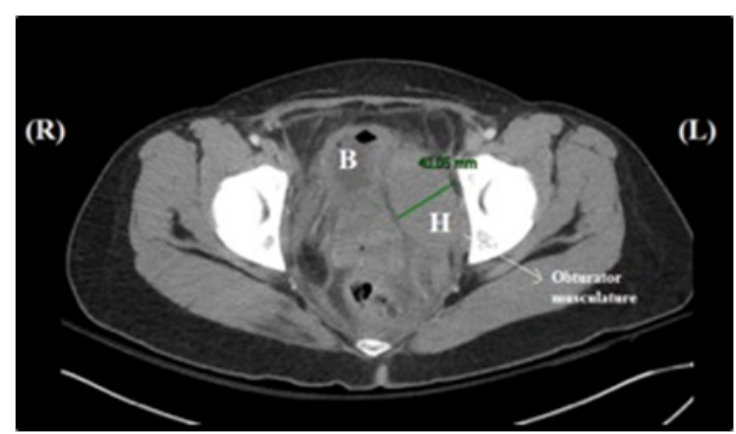

Figure 2 Transverse view of the obturator hematoma $(H)$ measuring $4.0 \mathrm{~cm}$ in width. The obturator musculature is lateral and posterior to the hematoma and the bladder $(B)$ is visualized. $(R)$, right; (L) left.

\section{Discussion}

Amongst the risks of the retropubic tape approach through the space of Retzius are bowel or vessel injury, bladder perforation and possible hematoma formation. The outside-in transobturator vaginal tape (TOT) procedure was subsequently developed to avoid damage to the bladder, bowel, and blood vessels within the vicinity of the space of Retzius. ${ }^{1}$ Postoperative complications associated with transobturator sling procedures include urinary retention, vaginal erosions, abscess formation, de novo dyspareunia, perineal pain and worsening of urgency. ${ }^{2}$

Vascular injury and subsequent hematoma formation are recognized, yet rare, complications of all sling procedures. Whiteside et al conducted cadaveric dissections in which they traced the regional vasculature and measured the distance from muscles, nerves and vessels to the transobturator sling. ${ }^{3}$ They found that after passing through the obturator canal, obturator vessels divided into medial and lateral branches that branched into smaller vessels at or near the end of the canal. ${ }^{3}$ In our patient's case, venous bleeding originating from these smaller vessels could have led to hematoma formation, likely limited by the tamponade effect of the confined region. The hematoma may have also been from suburethral bleeding and dissection with tracking along the tape, but unlikely as there was no acute bleeding at the end of the case.

Formation of hematomas in the pelvic region following sling procedures have been reported in the literature. Sun et al described a case of obturator hematoma measuring $8.1 \times 7.0 \times 5.1 \mathrm{~cm}$ after transobturator sling placement via inside-out transobturator vaginal tape approach (TVT-O) approach. ${ }^{4}$ Rajan and Kohli described cases of retropubic hematoma formation in patients who underwent transobturator sling placement. ${ }^{5}$ These authors noted that inadequate rotation of the needle of the trocar or pushing the needle too deeply into the region before rotation of the needle may extend the needle tip through the obturator internus and into the space of Retzius, resulting in vessel injury. ${ }^{5}$
Prompt recognition and management are crucial in the management of hematomas secondary to sling procedures. Hematoma formation in these cases have been evaluated by either CT scan or ultrasound. CT scan of the abdomen and pelvis provides a more accurate assessment of the hematoma location and is more useful if invasive intervention is required. ${ }^{4-6}$ Rajan and Kohli describe two cases of postoperative retropubic hematomas, one of which was managed conservatively with serial CT scans. ${ }^{5}$ Ultrasound has also been used to effectively manage these patients. The obturator hematoma described in Sun et al's second case was detected on both ultrasound and CT imaging measuring 14.5 x $10 \times 8.5 \mathrm{~cm}$ near the left obturator fossa compressing the bladder and was found to have resolved on repeat ultrasound five days after intraoperative ultrasound-guided drainage of the hematoma. ${ }^{6}$

Once the hematoma is identified on imaging, the patient's clinical condition dictates further management. As demonstrated by this case, patients who have minimal symptoms without signs of clinical deterioration can be observed irrespective of the location of the hematoma and followed with serial imaging. ${ }^{4-6}$

In summary, obturator hematomas after suburethral sling placement via the transobturator approaches are uncommon and usually self-resolve. Both CT and ultrasound of the abdomen and pelvis can provide the precise location and size of the hematoma. Patients who are clinically stable with minimal symptoms can be managed conservatively with pain control and serial imaging, if clinically indicated. ${ }^{6}$

\section{Acknowledgments}

None.

\section{Conflicts of interest}

The authors have no conflict of interest to declare.

\section{References}

1. Delorme E, Droupy S, Tayrac R, et al. Transobturator tape (uratape): a new minimally-invasive procedure to treat female urinary incontinence. European Urology. 2004;45:203-207.

2. Kaelin-Gambirasio I, Jacob S, Boulvain M, et al. Complications associated with transobturator sling procedures: analysis of 233 consecutive cases with a 27 months follow-up. BMC Women's Health. 2009;9:28.

3. Whiteside JL, Walters MD. Anatomy of the obturator region: relations to a trans-obturator sling. Int Urogynecol $J$ Pelvic Floor Dysfunct. 2004;15(4):223-226.

4. Sun MJ, Chen GD, Lin KC. Obturator hematoma after the transobturator suburethral tape procedure. Obstet Gynecol. 2006;108:716-8.

5. Rajan S, Kohli N. Retropubic hematoma after transobturator sling procedure. Obstet Gynecol. 2005;106:1199-1202.

6. Sun MJ.A life-threatening hematoma after the single-incision sling MiniArc procedure: a case report. J Minim Invasive Gynecol. 2013;20(4):529-532. 